\title{
Environmental Consciousness Among Graduation Students: Comparing Ukraine with Portugal
}

\section{Pedro Cosme Vieira}

Assistant Professor with Tenure and Habilitation, University of Porto, Portugal

\section{Yuliia Matvieieva}

$\mathrm{PhD}$, Senior Lecturer, Sumy State University, Ukraine

\section{Iuliia Myroshnychenko}

$\mathrm{PhD}$, Associate Professor, Sumy State University, Ukraine

\begin{abstract}
The present study was undertaken to evaluate and compare the environmental consciousness in Ukraine and Portugal and also to measure what is the impact of having this concept in the curricula of formal schooling, the sex and the age of the person.

To obtain micro data, as it is usual in the literature, we send a self-administered questionnaires by email to students of the Institute of Finance, Economics and Management of Sumy State University (Ukraine, Sumy) and to students of the Scholl of Economics and Management of the University of Porto (Portugal, Porto) from which we obtained 172 complete responses - 104 from Porto and 68 from Sumy.

We compute the Index of Environmental Consciousness and the Index of Schooling and we estimate a regression model using Weighted Linear Model from which were are able to conclude that 1) Portugal students have a significantly higher Environmental Consciousness than ones from Ukraine, 2) introducing the concept of Environmental Consciousness in the curricula of formal schooling has not significant impact on the Environmental Consciousness of students, 3) female people have a significantly higher Environmental Consciousness than the male ones and 4) age has a positive but not significant impact on the Environmental Consciousness of the person.
\end{abstract}

Using bootstrapping we investigate whether the assumption of a normal distribution of the error term would not cause catastrophic impact on estimators' t-statistics test and we concluded that results maintain valid.

We observe that the majority of people, $80.1 \%$, have environmental consciousness.

Keywords: ecological consciousness, sustainable development.

JEL Classification: Q56, C15, C83.

(C) The Authors, 2017. This article is published with open access at ARMG Publishing.

\section{Introduction}

It is well known that in a market economy where prices are the only incentive mechanism that guides the decisions of individuals, the "consumption of environment" (i.e., the negative impact of human activities in the environment) is just partially endogenized in the individual decision-making process of economic agents. We can argue that it is possible to design a contract where those that "consumer environment" will pay a price for it (i.e., a tax to the community) but to be perfect this procedure would need that transaction costs be low, Coase (1960), which is not verified as environment impact is difficult to observe and assess (e.g., notice the long discussion on the impact of human $\mathrm{CO}_{2}$ on "Climate Change") and it is transnational (e.g., if we discharge a pollutant into "our" ocean, all the ocean will become polluted). Being "consumption of environment" imperfectly endogenized in the decision-making process, it results that its consumption is higher than it would be socially optimal, i.e., mankind would be better off if individuals would adopt activities that would cause less negative impact on environment.

If the market mechanism induces human activities to have a higher than optimal impact on environment, one way of correcting this market failure is to use mass-media and the education system to introduce into the utility function of individuals the "consumption of environment" as a variable with negative weight, i.e., to increase in the mind of people the environmental consciousness / awareness (Stapp, 1969). 
Environmental consciousness is the tendency to reflect mentally on the environment and the impact of the individual behavior on it in a way that induces the individual to adopt more environmentally / ecologically conscious behavior (Antil, 1984; Shetzer et al., 1991). Environmental Consciousness /Awareness (ECA), is a change from the Dominant Social Paradigm, DSP, where people assume that resources are limitless, progress will resolve present day ecological problems and that there should always be a strict commitment to market competition (Catton and Dunlap, 1978; Dunlap and Van Liere, 1978) to a New Environmental Paradigm (NEP), where growth must be sustainable, i.e., without causing irreversible and significant environment impact (Roberts \& Bacon, 1997).

In the last decades, literature observes that an increasing number of consumers declare concerns about the impact of their actions on the environment and that this environmental consciousness impacts the decision making process (Wagner, 1997; Ottman, 1998). The problem is that, there is the common view among people that environmental consciousness implies that, by imposing limits to humanity's right to rule over the rest of nature, we are imposing limits to the growth of the economy: maintaining environmental quality implies, directly, the use of resources that leads to fewer resources being used on productive activities and, indirectly, obliges the use of less efficient technologies on productive activities. Although this percept negative linkage between environmental consciousness and economic growth, existing literature has diverse results regarding this connection being even possible that environmental friendly policies increase potential economic growth (Bovenberg \& de Mooij, 1997).

Most literature on the measurement of ECA uses distance self-reporting questioning or interviewing as primarily source of micro data. Althought self-reporting questionnaires has drawbacks (what people think or say they do is not necessarily what they actually think or do), direct measure of pro-environmental behaviour could not be considered in this research as it is very dificult and expensive( Dietz et al., 2005).

Harju-Autti \& Eevi (2014) measures national environmental awareness globally using online questionnaire to experts, which have relatively high environmental awareness and understanding of environmental matters in their own country and worldwide. In addition, respondents of each country had four other countries to assess. Similar to what we will do, Environmental Awareness Index, EAI, is computed as the arithmetic mean of General Education Indicator, Motivation to Act Indicator, and Personal Skills Indicator. The scale for every indicator (and consequently for the EAI) ranges from 0 to 100. Edmondson (2005) measures environmental consciousness using categorical scale (Likert scale from 1 to 5 ) that is similar to what we will use.

Being Environmental Consciousness / Awareness is a multidimensional concept, it is necessary to use more than one question to measure it. Kluckhohn (1962) assumes three basic components: Cognitive (knowledge); Affective (treatment) and Active (action) while Sanchez \& Lafuente (2010), surveying published theories of ECA, assume one more dimension - the Dispositional one. Sanchez \& Lafuente (2010) also aggregates several questions on an index but, instead of using the simple arithmetic mean, they compute weights for the each component by means of categorical principal components analysis.

Thompson \& Barton (1994) propose a "two motives" approach to ECA measuring; the ecocentrism (valuing nature for its own sake) and the anthropocentrism (valuing nature because of material or physical benefits it can provide for humans) that are computed from several questions as composite indexes.

Motivated by simplicity, we will use students as a proxy to a general population that is usual in the literature. For example, Selvam \& Nazar (2011) interview university students in India, and Tuna \& Özkoçak (2012) send questionnaires to university students in Turkey.

\section{Methodology}

To compare Portugal and Ukraine and identify the factors influencing the awareness of a person to perform certain actions towards nature, we carried out a survey among the students of the Institute of Finance, Economics and Management of Sumy State University (Ukraine, Sumy) and students of the Scholl of Economics and Management of the University of Porto (Portugal, Porto).

The questionnaire consisted of 14 questions divided into 4 groups.

Group 1-5 questions to measure Environmental Consciousness:

Q1 - Are you familiar with the standards and principles of sustainable development concept? (Yes; Partially; No). 
Q2 - What would stimulate you to improve complying with environmental norms? (I do not need any stimulus; A penalty; A reward).

Q3 - Do you believe that, by your action, you are able to improve the environment? (Yes; No).

Q4 - Do you believe that, by your example, is it possible to inspire others to change behavior, adopting actions that will improve the environment? (Yes; No).

Q5 - What would you do if you saw someone leaving trash outside containers? (I would personally put it in a container; I would make a comment; I would not pay attention).

These 5 questions intend to measure Motivation, Knowledge and Skills of respondent, Stapp (1969).

Group 2-2 questions to measure the inclusion of Environmental Consciousness on formal schooling:

Q6 - Did you have classes related to environmental education at high school? (Yes; No).

Q7 - Did you have or do you have classes related to environmental education at university? (Yes, No).

Group 3-3 questions to measure the factors that we intend to evaluate its impact on Environmental Consciousness / Awareness:

Q8 - Sex (Female; Male).

Q9 - Age (Numeric).

Q10 - Local (Sumy; Porto).

Group 4-4 questions without any special objective:

Q11 - Would you attend classes on environmental education (Several hypotheses)?

Q12 - Specify the need to improve the ecological situation in the city (On a scale of 0 to 10).

Q13 - Would you take part in environmental project (Several hypotheses)?

Q14 - Specify 1-3 variants of the causes of environmental pollution in cities (Several hypotheses).

We send the questioner by email on April 2017 (Sumy) and May 2017 (Porto) and we had 200 replies (28 missing Age), 121 (17 missing Age) from Porto and 79 (11 missing Age) from Sumy, 67\% women (66.3\% excluding those that missed Age) and 60.5\% from Porto (the same percentage excluding those that missed Age).

Table 1. Statistics of responses

\begin{tabular}{|l|c|c|}
\hline \multicolumn{1}{|c|}{ Indicators } & Sumy State University & University of Porto \\
\hline $\begin{array}{l}\text { Q1 - Percentage of students familiar with environmental princi- } \\
\text { ples, standards, concept of sustainable development. }\end{array}$ & $21.1 \%$ & $79.0 \%$ \\
\hline $\begin{array}{l}\text { Q2 - Percentage of students who does not require additional } \\
\text { incentives to comply with environmental standards. }\end{array}$ & $87.3 \%$ & $64.5 \%$ \\
\hline $\begin{array}{l}\text { Q3 - Percentage of students who believe that they personally } \\
\text { have the opportunity to improve the condition of the natural } \\
\text { environment. }\end{array}$ & $83.1 \%$ & $90.8 \%$ \\
\hline $\begin{array}{l}\text { Q4 - Percentage of students who believe that it is possible to } \\
\text { inspire others by own example to take care of the surrounding } \\
\text { nature. }\end{array}$ & $94.8 \%$ & $87.4 \%$ \\
\hline $\begin{array}{l}\text { Q5 - Percentage of students who would personally put in a } \\
\text { container trash leaved outside containers. }\end{array}$ & $30.4 \%$ & $57.4 \%$ \\
\hline $\begin{array}{l}\text { Q6 - Percentage of students who had classes connected with the } \\
\text { environmental education at school. }\end{array}$ & $80.5 \%$ & $45.40 \%$ \\
\hline $\begin{array}{l}\text { Q7 - Percentage of students who had classes connected with the } \\
\text { environmental education at the university. }\end{array}$ & $57.1 \%$ & $23.5 \%$ \\
\hline Q9 - Average the age of students (years). & 20.4 & 26.6 \\
\hline GDP per capita, current USD (2016, World Bank). & 2185.7 & 19813.3 \\
\hline
\end{tabular}

With the data obtained (172 observations), we intend to estimate an econometric regression mode. To be technically possible to do this, first, we transformed "Yes" in 1, "Partially" and "I would make a comment" in 0.5 and "No", "A penalty", "A reward" and "I would not pay attention" in zero. We also transformed "Female" and "Porto" in 1 and "Male" and "Sumy" in 0 (two dummy variables, D.Female and D.Porto). 
Second, we computed the Index of Environmental Consciousness, IECA = (Q1 + Q2 + Q3 + Q4 + Q5)/5 and the Index of Schooling, IS $=(\mathrm{Q} 6+\mathrm{Q} 7) / 2$. Assuming that the individual has Environmental Consciousness when the IEC is greater than or equal to 0.6 (i.e., answers Yes to at least 3 out of 5 questions), $72.4 \%$ of men and $87.7 \%$ of female (average of $80.1 \%$ ) has Environmental Consciousness.

Table 2. Comparing IECA e IS between Portugal and Ukraine (Welch Two Sample t-test)

\begin{tabular}{|c|c|c|c|c|c|c|c|}
\hline Variable & Global & Portugal & Ukraine & df & t-stat & p-value & Significant \\
\hline IECA & 0.73 & 0.76 & 0.69 & 169.8 & 2.55 & $1.2 \%$ & $5 \%$ \\
\hline IS & 0.50 & 0.37 & 0.70 & 142.2 & -5.83 & $0.0 \%$ & $0.1 \%$ \\
\hline
\end{tabular}

Third, assuming that sample is stratified, we computed two weighting factors, the first, related to the proportion of Females in the sample, wsex, W.Female $=1$ and W.Male $=66.3 \% /(1-66.3 \%)$, and the second related to the proportion of people from Porto in the sample, wplace, W.Porto $=1$ and W.Sumy $=60.5 \% /(1-60.5 \%)$.

Table 3. Comparing IECA e IS between Female and Male (Welch Two Sample t-test)

\begin{tabular}{|c|c|c|c|c|c|c|c|}
\hline Variable & Global & Female & Male & df & t-sat & p-value & Significant \\
\hline IECA & 0.73 & 0.76 & 0.67 & 87.3 & 2.44 & $1.7 \%$ & $5 \%$ \\
\hline IS & 0.50 & 0.55 & 0.39 & 112.8 & 2.58 & $1.1 \%$ & $5 \%$ \\
\hline Age & 24.1 & 22.9 & 26.5 & 79.5 & -2.70 & $0.8 \%$ & $1 \%$ \\
\hline
\end{tabular}

Finally, we become able to estimate the following regression model:

$\mathrm{IEC}=\mathrm{Intercept}+\beta 1 . \mathrm{IS}+\beta 2$.D.Female $+\beta 3$.D.Porto $+\beta 4$.Age + error.

We computed 4 models by using the function $\operatorname{lm}(\ldots)$ in the R program. 1) The "Simple Model" where all observations have the same weight; 2) the "Weighted model F" where the weight factor related to Sex; 3 ) the "Weighted model P" where the weight factor related to Place and 4) the "Weighted model F.P" where the weight factor related to both the Sex and the Place.

\#\#Program used in the estimation of the econometric models

Data.file <- read.csv("Data_total.csv") \#Reading data from file

Model.F.P <- $\operatorname{lm}\left(\right.$ IECA $~$ IS + D.Female + D.Porto + Age, Data.file, weight $=$ wsex ${ }^{*}$ wplace $)$

Model.P <- $\operatorname{lm}($ IECA $~$ IS + D.Female + D.Porto + Age, Data.file, weight $=$ wplace $)$

Model.F <- $\operatorname{lm}($ IECA IS + D.Female + D.Porto + Age, Data.file, weight = wsex $)$

Model.Simple <- $\operatorname{lm}($ IEC $~$ IS + D.Female + D.Porto + Age, Data.file $)$

summary(Model.F.P) \#To obtain results. Similar to other models.

In all 4 models, parameters associated with the variables IS and Age are not significant. Parameters associated with Female and Porto are positive and significant at $1 \%$ and 5\%, respectively.

Table 4. Average of all 4 models, significance computed from t-statistics

\begin{tabular}{|c|c|c|c|c|}
\hline Variable & Estimate & t-stat & Probability & Significance \\
\hline (Intercept) & 0.514 & 7.527 & $0.00 \%$ & $0.1 \%$ \\
\hline School & 0.023 & 0.502 & $63.05 \%$ & N.S. \\
\hline D.Female & 0.114 & 3.262 & $0.14 \%$ & $5 \%$ \\
\hline D.Porto & 0.083 & 2.034 & $4.52 \%$ & NS \\
\hline Age & 0.003 & 1.396 & $16.56 \%$ & \\
\hline $\mathrm{R}^{2}$ & $9.42 \%$ & & & \\
\hline
\end{tabular}

Due to the fact that the variables are categorical, there is a methodological doubt on the use of the t-statistic to evaluate the significance of the parameters. To overcome this doubt, we conducted estimation by the bootstrapping method in which we re-sampled with reposition the original data 25000 times (a total of 100000) and we used obtained estimatins to compute the significance (the p-value is the percentage of times that the estimative is smaller than or equal to zero (when mean is positive). We used the following $\mathrm{R}$ program (just for the "Weighted model F.P", the others' code is very similar). 
\#Bootstrapping R program - Weighted model F.P

$\mathrm{N}=172$ \#Number of observations in the original data

Zeros $=\operatorname{rep}(0,25000)$

results $<$ - data.frame $($ Intercept $=$ Zeros, School $=$ Zeros, D.Fem $=$ Zeros, D.Porto $=$ Zeros, Age $=$ Zeros $)$

for (i in 1:25000) \#Number of re-samplings

$\{$ training $=$ sample $(1: \mathrm{N}, \mathrm{N}$, replace $=$ TRUE $) \#$ Re-sampling with reposition

1.data $=$ Data.file[training, $\#$ Data.file is the original data

model <- $\operatorname{lm}($ IECA $\sim$ IS + D.Female + D.Porto + Age, 1.data, weight $=$ wsex $*$ wplace $)$

results[i, $]=$ model $\$$ coefficients[1:5] $\}$

for (i in 1:5)

$\operatorname{print}(\operatorname{nrow}($ results [results[,i] $<=0]) / 25000$,

The advantage of using of bootstrapping method it is that it is not necessary to make any conjecture on the distribution of errors. By using this methodology, we observe that results on significance of variables are very similar with those that we obtained using t-statistics that indicates that its use is acceptable with our data.

Table 5. Average of 4 models using bootstrapping ( 4 x 25000 iterations)

\begin{tabular}{|c|c|c|c|}
\hline Variable & Estimate & Probability & Significant \\
\hline (Intercept) & 0.513 & $0.00 \%$ & $0.1 \%$ \\
\hline School & 0.023 & $31.6 \%$ & NS \\
\hline D.Female & 0.114 & $0.12 \%$ & $1 \%$ \\
\hline D.Porto & 0.083 & $2.1 \%$ & $5 \%$ \\
\hline Age & 0.003 & $10.1 \%$ & NS \\
\hline
\end{tabular}

Within the research we finished the questionnaire with 5 questions related with students environment awareness but with no special meaning.

In question Q11, we asked students under what conditions the person would receive additional knowledge on environmental education. The majority of students would receive environmental knowledge only if it would be free or they would have a small payment for what $(39.2 \%+10.8 \%$ at Sumy and $44.6 \%+10.1 \%$ at Porto).

In question Q12, we asked students to assess the necessity of improvement of the condition of the natural environment (CNE) in their native city on a scale from zero (no need for improvement) to ten (maximum need of improvement). Most students, 88\% answer 5 or bigger, both of the Institute of FEM (average of 7.7) and of the FEP of the University of Porto (average of 6.4), considering that it is necessary to improve the condition of the natural environment in their home city (see Figure 1 in Appendix). It is interesting to notice that, although Ukraine students have smaller ECA, they indicate a stronger need of improvement in their home city. Being the scores so high, it indicates students identify serious environmental problems both in Ukraine and Portugal cities.

In question Q13, to identify the motivation of students for ecologically-oriented behavior, we asked in which case the students would take part in the environmental project. The students of SSU said they would do it: On their own initiative $(72.2 \%)$ and; In case they would receive a reward (22.2\%) while students of the FEP of the University of Porto would do it: On their own initiative (50\%) and; In case they would receive a reward $(41.4 \%)$.

In question Q14, the students identified the most common causes of natural environment pollution. Those from the Sumy State University identified that: People are convinced that the caused pollution won't hurt them personally (33.8\%); lack of ecological culture and education (28.6\%); imperfect system of punishment (10.4\%); imperfect incentive system (6.5\%) and; people are convinced that the caused damage is insignificant $(3.9 \%)$ are the principal factors while those from FEP of the University of Porto defined that: people 
are convinced that their influence is insignificant (63.9\%); people don't have ecological culture (60.5\%); people are convinced that pollution won't hurt them personally (52.9\%); imperfect system of punishment (41.2\%); people do not know that their personal actions influence the level of pollution (37.8\%) and; Imperfect incentive system (17.6\%) are the principal causes of natural environment pollution.

It sums up more than $100 \%$ due to the fact that students had the opportunity to give several answers to this question.

\section{Conclusions}

In a market economy where equilibrium "environment" price is zero, people on an individual basis cause higher negative impact on environment than would be socially optimal. One way of correcting this market failure is to increase the Environmental Consciousness and Awareness (ECA), on people that will induce them to adopt environment friendly consumption decisions not uniquely based on market prices. In this paper, we intend to evaluate and compare the ECA on Ukraine and Portugal and also to measure what is the impact of having this concept in the curricula of formal schooling, sex and age of the person.

We carried out a survey among students of the Institute of Finance, Economics and Management of Sumy State University (Ukraine, Sumy) and students of the Scholl of Economics and Management of the University of Porto (Portugal, Porto). With 172 complete responses, 104 from Porto and 68 from Sumy, we were able to estimate an econometric model from which we could conclude that:

H1) Portugal people have a significantly higher Environmental Consciousness than people from Ukraine.

$\mathrm{H} 2$ ) Introducing the concept of EC in the curricula of formal schooling has not significant impact on the EC of students.

H3) Female people have a significantly higher Environmental Consciousness than male people.

H4) Age has a positive impact on the Environmental Consciousness of the person but results are not significant (but they are almost significant at the $10 \%$ threshold).

Due to the fact that we use categorical data both in the dependent variable (the Index of Environmental Consciousness) and in the independent variables (the Index of Schooling, country, sex and age), there was doubt on the use of the t-statistic because its validity is dependent on the errors having normal distribution. To investigate this question, we estimated the regression models using Bootstrapping (re-sampling) and we concluded that statistical results continue valid.

We observe that the majority of people, $80.1 \%$, have Environmental Consciousness that is in accord with the literature (e.g., Samdahl \& Robertson, 1989).

Finally, the conclusion that Portuguese have a significantly higher ECA than people from Ukraine in accordance with literature that reflects differences in the GDP per capita, higher in Portugal.

\section{References}

1. Antil, John H. (1984). Socially responsible consumers: profile and implications for public policy. Journal of Macromarketing, 4, 18-39.

2. Bovenberg, Lans and Ruud de Mooij (1997). Environmental tax reform and endogenous growth. Journal of Public Economics, 63(2), 207-237.

3. Catton, William R., and Riley E. Dunlap (1978). Environmental sociology: a new paradigm. American Sociologist, 13, 41-49.

4. Coase, Ronald H. (1960). The problem of social cost. Journal of Law \& Economics, 3, 1-44.

5. Dembkowski, S. and S. Hanmer-Lloyd, S. (1994). The environmental value-attitude-system model: a framework to guide the understanding of environmentally-conscious consumer bahaviour. Journal of Marketing Management, 10(7), 593-603.

6. Dietz, T., Fitzgerald, A. \& R. Shwom, R. (2005). Environmental values. Annual Review of Environmental Resources, 30, 335-372.

7. Dunlap, Riley E., and Kent D. Van Liere (1978). The New Environmental Paradigm. Journal of Environmental Education, 9, 10-19.

8. Edmondson, D. R. (2005). Likert scales: a history. Proceedings of CHARM - the Conference on Historical Analysis and Research in Marketing, California, USA, 12. Available at 
http://faculty.quinnipiac.edu/charm/CHARM\%20proceedings/CHARM\%20article\%20archive \%20pdf\%20format/Volume\%2012\%202005/1 27\%20edmondson.pdf.

9. Harju-Autti, Pekka and Kokkinen Eevi (2014). A Novel Environmental Awareness Index Measured Cross-Nationally For Fifty Seven Countries. Universal Journal of Environmental Research and Technology, 4(4), 178-198.

11. Ottman, Jacquelyn A. (1998). Green marketing: opportunity for innovation, 2nd edition, pp. 1-270. NTC Business Books: Chicago.

12. Kluckhohn, Clyde (1962). Culture and Behavior, Free Press of Glencoe.

13. Roberts, James A. and Donald R. Bacon (1997). Exploring the subtle relationships between environmental concern and ecologically conscious consumer behavior. Journal of Business Research, 40(1), 79-89.

14. Samdahl, D. M., and R. Robertson (1989). Social determinants of environmental concern: Specification and test of the model. Environment and Behavior, 21, 57-81.

15. Sanchez, M. J. and R. Lafuente (2010). Defining and measuring environmental consciousness. Revista Internacional de Sociología (RIS), 68(3), 731-755.

16. Selvam, V. and N. Abdul Nazar, N. (2011). An analysis of environmental awareness and responsibilities among university students. International Journal of Current Research, 11(3), 202-205.

17. Shetzer, L., Stackman, R. W. and L. F. Moore, (1991). Business-environment attitudes and the new environmental paradigm. Journal of Environmental Education, 22(4), 14-21.

18. Stapp, W. B., D. Bennett, W. Bryan Jr., J. Fulton, J. MacGregor, P. Nowak, J. Swan, R. Wall, and S. Havlick (1969). The concept of environmental education. Journal of Environmental Education, 1(1), 30-31.

19. Thompson, S., Gagnon, C. and Barton, A., Michelle, A. (1994). Ecocentric and anthropocentric attitudes toward the environment. Journal of Environmental Psychology, 14(2), 149-157.

20. Tuna, Y. and Özkoçak, L. (2012). The first step to communication with environmentally responsible consumer: measuring environmental consciousness of Turkish consumers. Online Journal of Communication and Media Technologies, 2(3).

21. Wagner, S. A. (1997). Understanding green consumer behavior: a qualitative cognitive approach. Routledge, 1-304.

\section{Appendix}

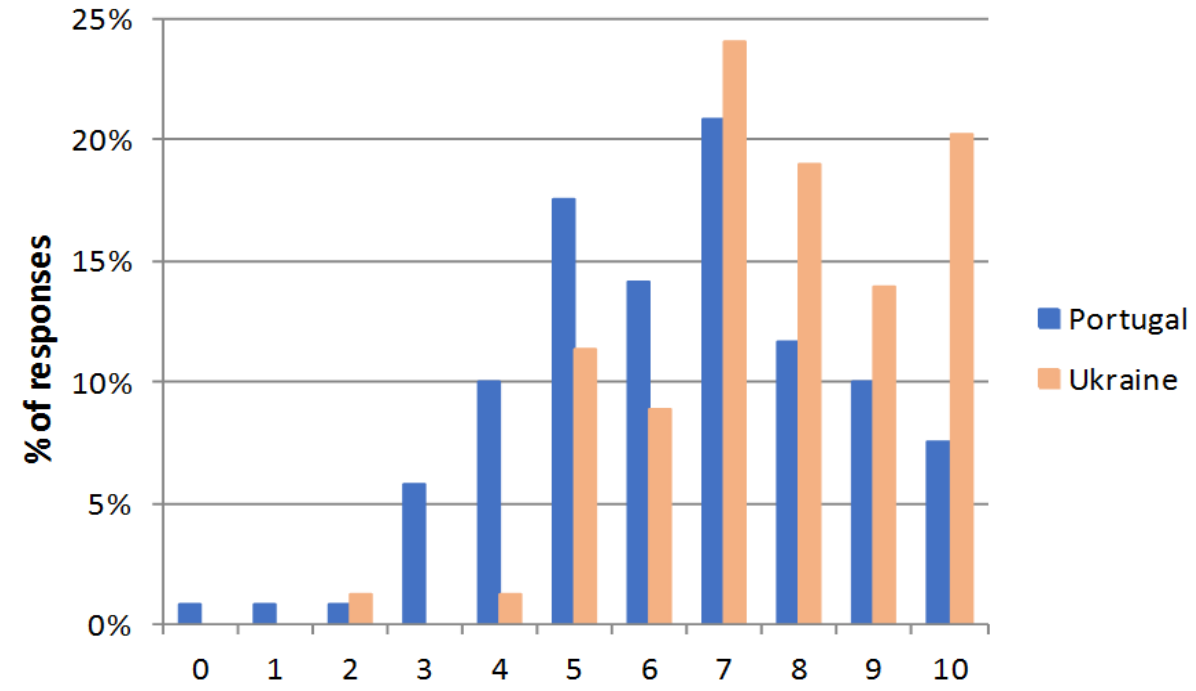

Figure 1. Responses to Q12 - Specify the need to improve the ecological situation in the city 